\title{
The dynamics of the vertical structure of turbulence in flood flows
}
D. J. Georgiev ${ }^{1}$
A. J. Roberts ${ }^{2}$
D. V. Strunin ${ }^{3}$

(Received 31 August 2006; revised 28 June 2007)

\begin{abstract}
The flow of water in a tide, flood or tsunami is often turbulent. We model large scale, but shallow, turbulent flow using the well established $k-\omega$ model of turbulence. Vertical turbulent mixing underlies the existence of a slow manifold model. As a first step, in this article the flow is assumed laterally homogeneous. Then constructing the slow manifold discovers the evolution of the average lateral velocity and average turbulent energy. We focus upon the influence on the mathematical analysis of key physical factors affecting the dynamics: turbulent mixing, energy production due to shear, volume energy dissipation and gravitational forcing on sloping ground. Further research will incorporate large scale lateral variations in the flow in order to predict tides, floods and tsunamis.
\end{abstract}

See http://anziamj.austms.org.au/ojs/index.php/ANZIAMJ/article/view/124 for this article, (C) Austral. Mathematical Soc. 2007. Published December 7, 2007. ISSN 1446-8735 


\section{Contents}

1 Seek a turbulent model of shallow water

C574

2 Numerical quasi-balance indicates direction

C576

3 A slow manifold model

C582

4 Conclusion

C586

References

C587

\section{Seek a turbulent model of shallow water}

We aim to model turbulent shallow water flows such as floods, tsunamis and the tidal bores shown in Figure 1. Such flows have important effects upon our environment [1, 10, e.g.]. Quantities of prime interest are: the fluid depth at position $x$ and time $t, \eta(x, t)$; the mean lateral velocity $\bar{u}(x, t)$; the mean turbulent kinetic energy $\bar{k}(x, t)$; and the mean rate of turbulent dissipation $\bar{\omega}(x, t)$. But, averaging of dynamical equations is unsound [11, p.153, e.g.] as sometimes done for turbulent flow [6, 7, 9, e.g.]; instead, centre manifold theory $[14,15]$ puts such dynamical models on a firm basis. However, before modelling the dynamics of lateral variations in wave equations of the form

$$
\frac{\partial \eta}{\partial t}=-\frac{\partial}{\partial x}(\bar{u} \eta), \quad \frac{\partial \bar{u}}{\partial t}=-\bar{u} \frac{\partial \bar{u}}{\partial x}+g \times \text { slope }-\operatorname{drag}, \quad \ldots,
$$

such as equations (14)-(16), we must first model the dynamics of the vertical structure of such turbulent flows. This first step is the topic of this article. The aim is to develop the mathematical analysis necessary to construct sound computational models of turbulent floods, tsunamis and tides. 


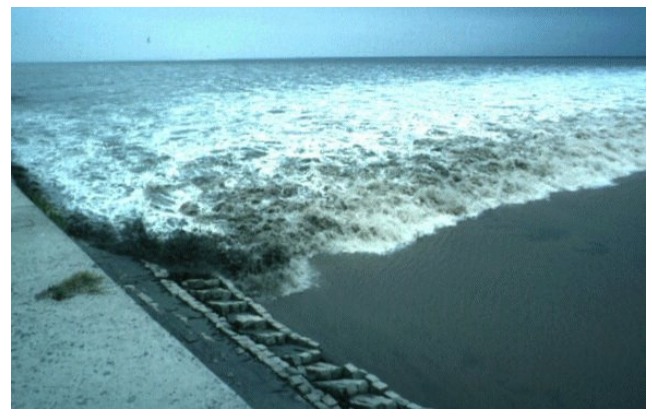

(a) Qiantang River

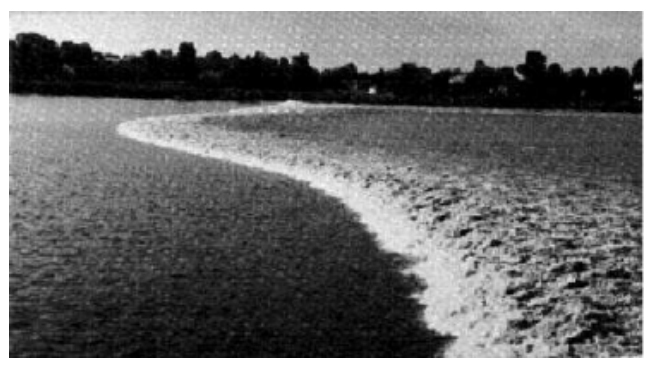

(b) Severn River

Figure 1: two tidal bores display very high turbulence: (a) http://www. uq.edu.au/ e2hchans/pictures/hang02.jpg by Eric Jones; (b) http://www. efluids.com/efluids/gallery/gallery_pages/hydraulic_jump_page.htm by D. H. Peregrine.

The model is to be expressed in terms of vertically averaged quantities. The alternative of resolving the vertical structure of the turbulent flow is, as noted by Gross [8], "computationally demanding" and "generally some compromise is sought in the modeling approach". Gross continues [8]: "One commonly used approach for reducing computational effort is to depth-average, ... However, if important mechanisms are not resolved by a depth-averaged approach, a 3D model may nonetheless be required for simulations".

In contrast, we do not depth-average the equations. Instead we use dynamical systems theory to resolve flows with significant vertical structure, yet do so expressing the model in terms of averaged quantities. For example, Roberts and Mercer $[12,17]$ faithfully modelled the shear dispersion in flow along varying pipes; the same methodology will here analogously account for vertical dynamics that affect lateral modeling. By modelling turbulent intensity we aim to encompass flows that range from highly turbulent to effectively laminar irrotational dynamics. Our aim is to capture the critical structures of the vertical dynamics of turbulent floods, equations (11)-(13), while maintaining the relative simplicity and computational tractability in 
modelling the flows in terms of only averaged quantities.

Here we ignore horizontal variations, $\partial_{x}=0$, and horizontal pressure gradients. Instead, we investigate the vertical profile across the flow in $0<$ $z<\eta: u(z, t)$ is the horizontal velocity at height $z$ above the ground; $k(z, t)$ is the turbulent kinetic energy; and $\omega(z, t)$ is the rate of turbulent dissipation. Vertical turbulent mixing rapidly smoothes the vertical profiles. Thereafter, a quasi-balance occurs in the evolution to equilibrium between forcing and bed drag. We use the numerics of Section 2 and the centre manifold theory of Section 3 to find the quasi-balance of the vertical profiles and their consequent long term evolution. Then we validate the modelling of vertical dynamics by the prediction of uniform channel flow, equation (17), and a power law turbulent decay, equation (18).

\section{Numerical quasi-balance indicates direction}

For high Reynolds number turbulent flow relevant to floods, we explore Wilcox's $k-\omega$ model [18] as it often performs well near boundaries [5]. With eddy viscosity $\nu=k / \omega$, and laminar viscosity neglected, Wilcox's model is

$$
\begin{aligned}
& \frac{\partial u}{\partial t}=\frac{\partial}{\partial z}\left(\nu \frac{\partial u}{\partial z}\right)+\gamma g_{x}-\mu_{u} \\
& \frac{\partial k}{\partial t}=\frac{1}{2} \frac{\partial}{\partial z}\left(\nu \frac{\partial k}{\partial z}\right)+\nu\left(\frac{\partial u}{\partial z}\right)^{2}-\frac{9}{100} \gamma \omega k-\mu_{k} \\
& \frac{\partial \omega}{\partial t}=\frac{1}{2} \frac{\partial}{\partial z}\left(\nu \frac{\partial \omega}{\partial z}\right)+\frac{5}{9}\left(\frac{\partial u}{\partial z}\right)^{2}-\frac{3}{40} \gamma \omega^{2}-\mu_{\omega}
\end{aligned}
$$

when the parameters $\gamma=1$ and $\mu_{u}=\mu_{k}=\mu_{\omega}=0$. The purpose of the artificial parameter $\gamma$ and the 'Lagrange multipliers' $\boldsymbol{\mu}=\left(\mu_{u}, \mu_{k}, \mu_{\omega}\right)$ will be 
explained in due course. We use the following boundary conditions on the free surface and the ground:

$$
\begin{aligned}
& \frac{\partial u}{\partial z}=\frac{\partial k}{\partial z}=\frac{\partial \omega}{\partial z}=0 \quad \text { on } \quad z=\eta ; \\
& u=14.77 u_{*}, \quad k=4.78 u_{*}^{2}, \quad \omega=81.5 u_{*} / \eta \quad \text { on } \quad z=0,
\end{aligned}
$$

where the 'friction velocity' $u_{*}=\sqrt{\nu u_{z}}$ at $z=0$. The ground boundary conditions (5) apply for the inertial sub-layer where the viscous effects are small in comparison with those of turbulence: this is the "hydraulically rough" case [8, p.1200]. In this first exploration of this dynamical systems approach, although we do not implement boundary conditions on the turbulence analogous to those suggested by Blumberg et al. [3, 2], we do use ground and free surface boundary conditions on the velocity analogous to those implemented by Gross [8]. The ground and free surface conditions (4)-(5) ensure that predicted profiles for fully developed channel flow reasonably match the experimental profiles summarised by Nezu [13].

This section constructs a three dimensional set of states, called the 'adiabatic manifold', in $(u(z), k(z), \omega(z))$ space which contains all the relatively slow evolution of the dynamics of the model (1)-(3). This adiabatic manifold is parametrised by the depth averaged 'amplitudes' $(\bar{u}, \bar{k}, \bar{\omega})$. The vertical structures we find approximate the quasi-balance of vertical mixing.

But how can we find a manifold of slow evolution? We want to specify some fixed arbitrary $(\bar{u}, \bar{k}, \bar{\omega})$ as input parameters. But then for almost all $(\bar{u}, \bar{k}, \bar{\omega})$ the turbulent equations (1)-(3) cannot be an equilibrium. However, now consider the artificially introduced 'Lagrange multipliers' $\boldsymbol{\mu}$ in (1)-(3): allowing them to vary, they provide the freedom to find quasi-equilibrium solutions of the turbulent equations (1)-(3).

Thus, to construct this adiabatic manifold, first set to zero the time derivatives on the left-hand side of (1)-(3). Then solve these turbulence equations for equilibria, including Lagrange multipliers $\boldsymbol{\mu}$ as variables $(\gamma=1$ in this section), with the constraint that the turbulent averages $(\bar{u}, \bar{k}, \bar{\omega})$ 
are fixed. In essence, this task is a nonlinear eigenvalue problem with potentially many solutions; we seek the solution with smallest parameters $\boldsymbol{\mu}$. Repetitively find these equilibria over a representative range of turbulent averages $(\bar{u}, \bar{k}, \bar{\omega})$; typically we sought solutions on a uniform $15^{3}$ lattice in $(\bar{u}, \bar{k}, \bar{\omega})$ space. Second, realise that each found solution gives profiles $(u(z), k(z), \omega(z))$ for the corresponding turbulent flow. Substitute these profiles into Wilcox's turbulent model (1)-(3), set the Lagrange multipliers $\boldsymbol{\mu}=\mathbf{0}$ to give physical equations, and then average over the fluid depth to deduce the time evolution of the turbulent averages $(\bar{u}, \bar{k}, \bar{\omega})$. But the averages of the right-hand sides are precisely the Lagrange multipliers $\boldsymbol{\mu}$. Thus the slow evolution predicted by this adiabatic manifold is

$$
\frac{d \bar{u}}{d t}=\mu_{u}, \quad \frac{d \bar{k}}{d t}=\mu_{k}, \quad \frac{d \bar{\omega}}{d t}=\mu_{\omega} .
$$

These ODEs form a closed set of equations as the Lagrange multipliers $\boldsymbol{\mu}$ are, by construction, now known functions of $(\bar{u}, \bar{k}, \bar{\omega})$. Thus this numerical construction discovers a comprehensive range of states of quasi-balance in the vertical structure of turbulence, and predicts the long term evolution (6) between these states. ${ }^{1}$

A preliminary implementation of the above algorithm finds turbulent flow solutions at about $63 \%$ of 3,375 lattice points ${ }^{2}$ for the part of the domain of interest. Difficult parameter regimes are low turbulent energy $\bar{k}$, low $\bar{\omega}$, and high flow velocity $\bar{u}$; these regions are missing from the results shown in Figures 2-4.

Figures 2-4 tentatively plot isosurfaces of the three Lagrange multpliers over the domain. The variables are non-dimensionalised with respect to the length scale of the depth of the fluid $\eta$, the velocity scale $\sqrt{g_{x} \eta}$ and hence the time scale $\sqrt{g_{x} / \eta}$. The intersection of the null-surface for each of the

${ }^{1}$ The spectrum of the dynamical system linearised about each point on an adiabatic manifold should then give the rate of attraction to the adiabatic manifold. There should be a significant spectral gap, but as yet we have not explored this aspect.

${ }^{2}$ The computation took about 20 minutes CPU on a desktop Linux computer. 


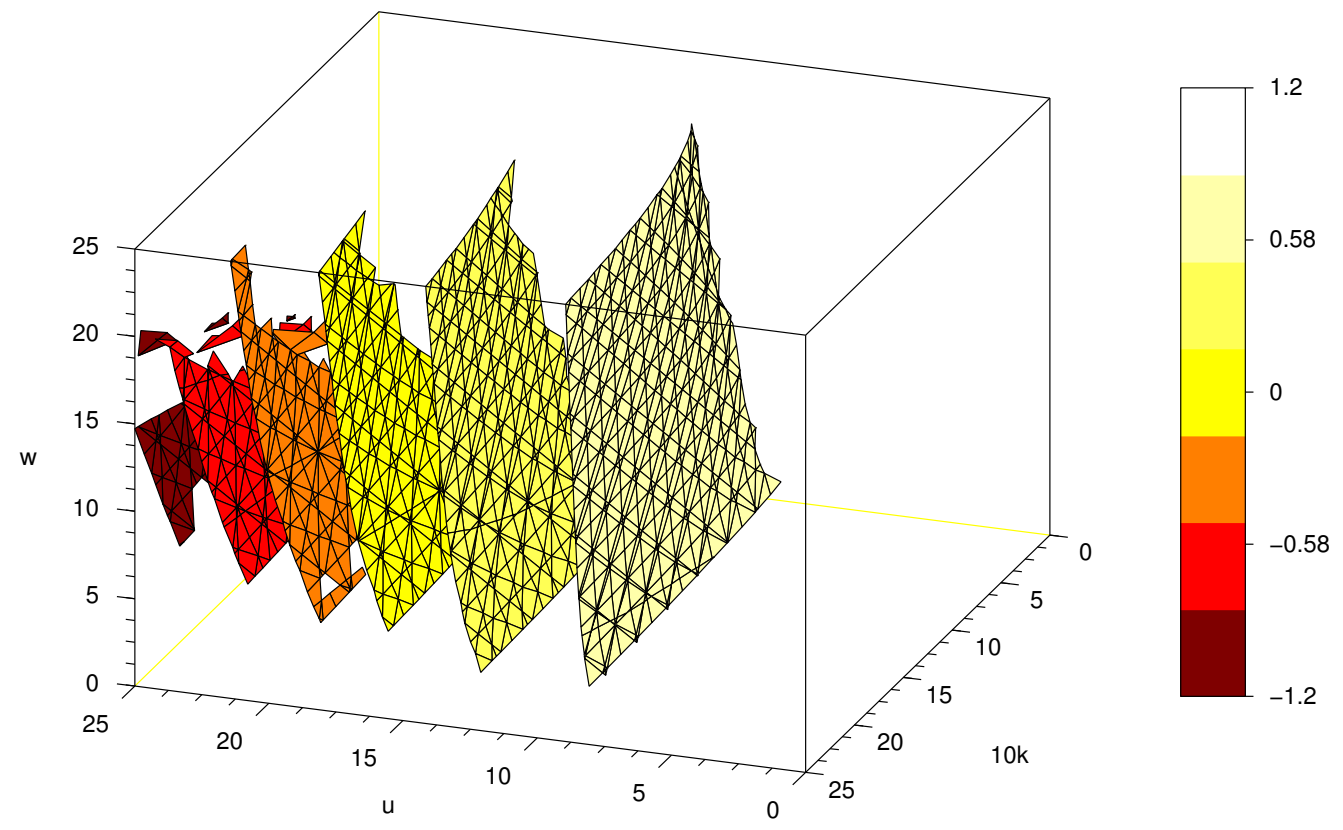

FIGURE 2: isosurfaces of the rate $\mu_{u}$ that the mean lateral velocity $\bar{u}$ evolves as a function of non-dimensional $(\bar{u}, \bar{k}, \bar{\omega})$ (the $\bar{k}$ axis is stretched by a factor of ten). 


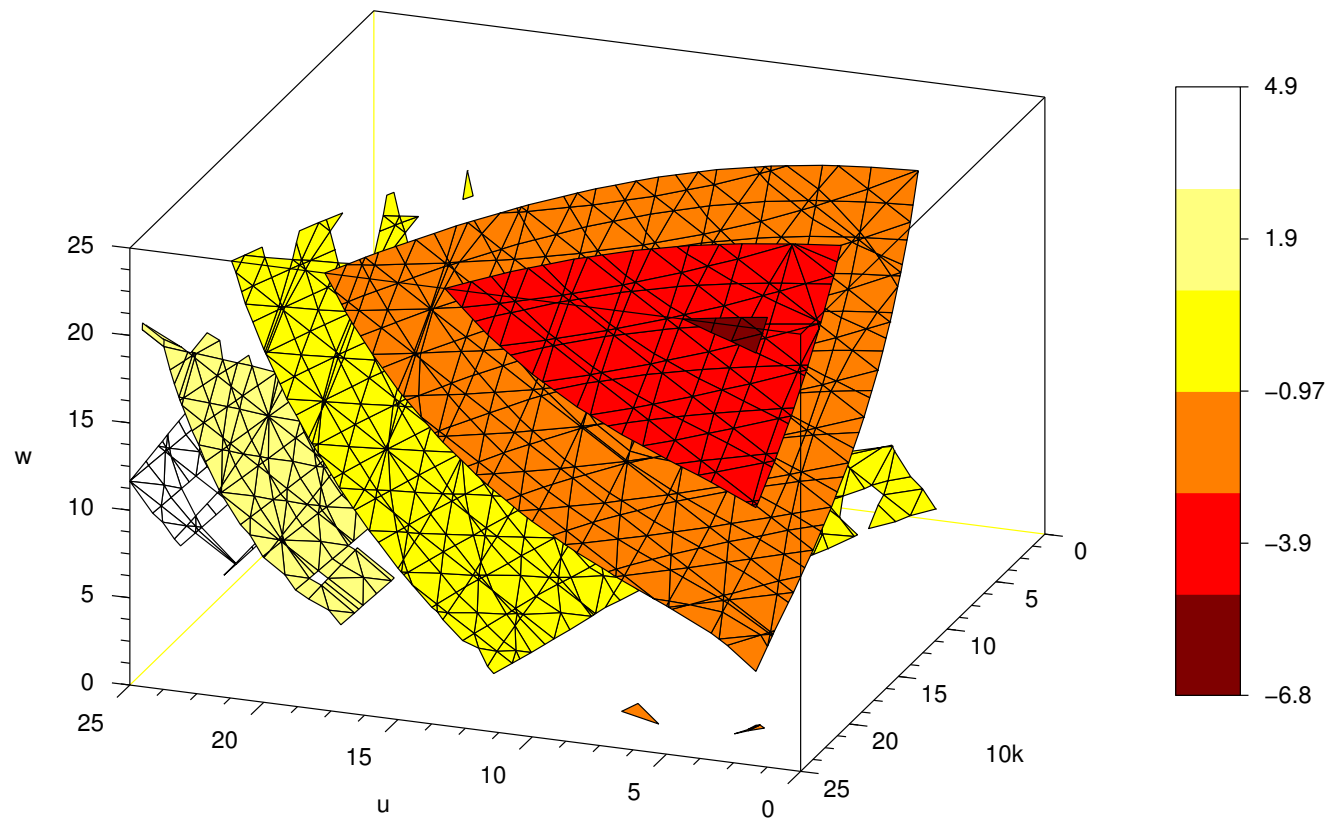

FiguRE 3: isosurfaces of the rate $\mu_{k}$ that the mean turbulent energy $\bar{k}$ evolves as a function of non-dimensional $(\bar{u}, \bar{k}, \bar{\omega})$ (the $\bar{k}$ axis is stretched by a factor of ten). 


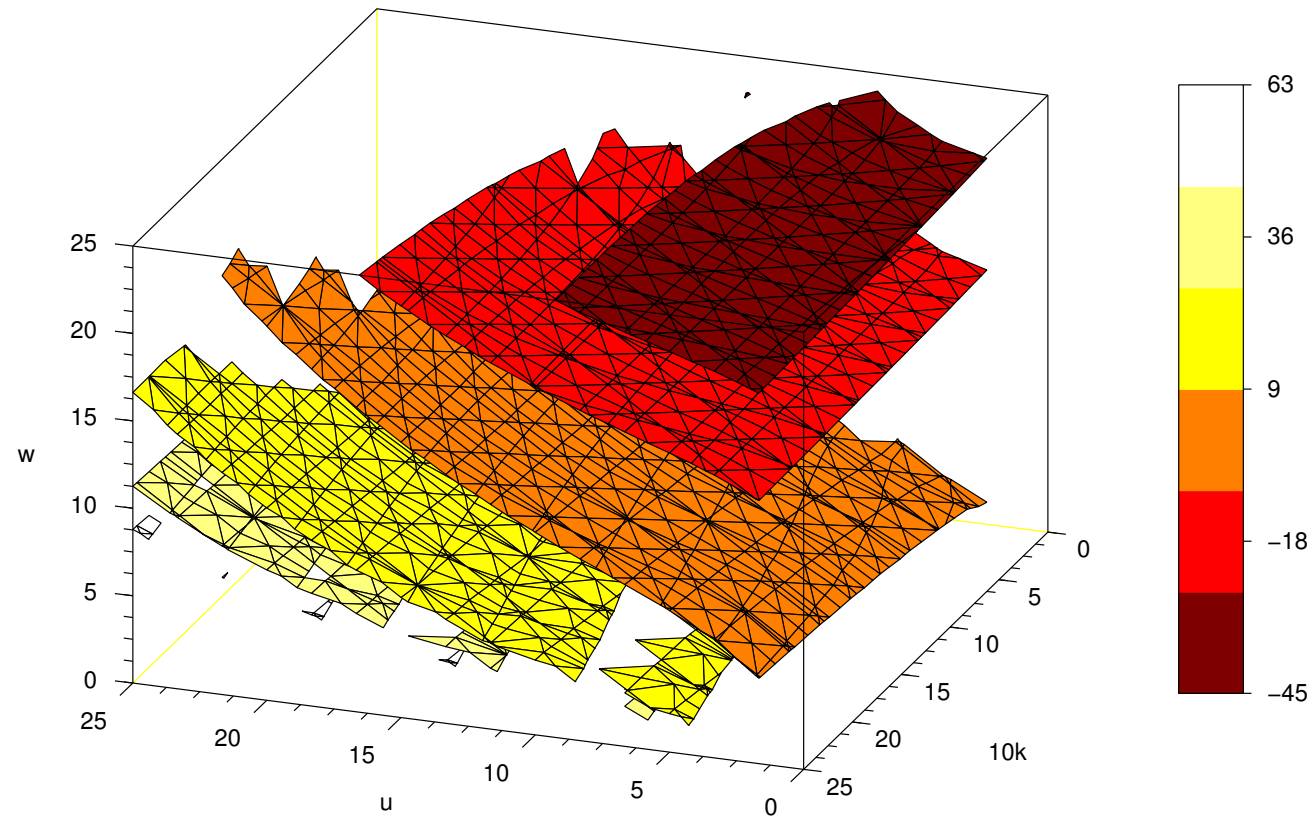

FigURE 4: isosurfaces of the rate $\mu_{\omega}$ that the turbulent mean $\bar{\omega}$ evolves as a function of non-dimensional $(\bar{u}, \bar{k}, \bar{\omega})$ (the $\bar{k}$ axis is stretched by a factor of ten). 
three fields, then predicts mean turbulent properties of uniform channel flow, namely

$$
\bar{u}=18.3 \sqrt{g_{x} \eta}, \quad \bar{k}=1.93 g_{x} \eta, \quad \bar{\omega}=19.1 \sqrt{g_{x} / \eta} .
$$

This velocity $\bar{u}$ matches the well known experimentally determined drag coefficient, $c_{D}=0.003$, in the Chezy law for fully developed channel channel flow [9, e.g.]. This is only one prediction. We seek models for the time evolution of turbulent flow.

But also note that the $\omega$ field evolves much faster than $k$ and $u$, in the plotted domain as seen in the colour bars: the non-dimensional rate $\mu_{\omega}$ ranges over about $[-40,80]$; the rate $\mu_{k}$ ranges over about $[-8,4]$; and the rate $\mu_{u}$ ranges over about $[-1,1]$. Thus expect the mean $\bar{\omega}$ to reach approximate equilibrium an order of magnitude faster than the mean turbulent energy $\bar{k}$ and the mean flow $\bar{u}$. Consequently, perhaps we should look for a model that resolves the dynamics of mean turbulence and flow, $\bar{k}$ and $\bar{u}$, but treats all of the turbulent $\omega$ as slaved. However, for the moment persevere with modelling with all three coarse variables $\bar{u}, \bar{k}$ and $\bar{\omega}$.

\section{A slow manifold model}

We proceed to develop an analytic model that approximately captures the dynamics of the previous numerical exploration. The approximate slow manifold is constructed algebraically in order to arrive at an algebraic model for laterally uniform turblent flow. The model is expressed in terms of the evolution of mean quantities $\bar{u}, \bar{k}$ and $\bar{\omega}$.

Modified ground conditions ensure a slow manifold We seek to satisfy the ground boundary conditions (5) from an artificial equilibria of uniform turbulent properties. Thus absorb the ground boundary conditions (5) 
into the following parametrised versions:

$$
\begin{array}{ll}
(1-\gamma) \frac{\partial u}{\partial z} & =\gamma\left[u^{2}-15^{2} \nu \frac{\partial u}{\partial z}\right] \frac{6}{11 \eta \bar{u}} \quad \text { on } z=0 ; \\
(1-\gamma) \frac{\partial k}{\partial z} & =\left[\gamma k-\frac{19}{4} \nu \frac{\partial u}{\partial z}\right] \frac{\eta \bar{\omega}^{2}}{8 \bar{k}} \quad \text { on } z=0 ; \\
(1-\gamma) \frac{\partial \omega}{\partial z} & =\left[\gamma \omega^{2}-\frac{81^{2}}{\eta^{2}} \nu \frac{\partial u}{\partial z}\right] \frac{\eta \bar{\omega}}{113 \bar{k}} \quad \text { on } z=0 .
\end{array}
$$

The artificial parameter $\gamma$ interpolates between a useful analytic base, when $\gamma=0$, to physically reasonable ground boundary conditions when $\gamma=1$. We base the analytic analysis on the case $\gamma=0$, construct power series solutions in $\gamma$, and seek results for the physical case $\gamma=1$.

Theory asserts there exists a slow manifold model. Ignore the artificial Lagrange multipliers $\boldsymbol{\mu}$ and seek to solve Wilcox's turbulent equations (1)-(3), with the free surface slip boundary conditions (4), and the ground boundary conditions (8)-(10). When $\gamma=0$ the right-hand sides in the ground boundary conditions (8)-(10) vanishes so that a three dimensional centre space of equilibria exist, namely the tubulent fields are constant in the vertical: $u=\bar{u}, k=\bar{k}$ and $\omega=\bar{\omega}$. Then centre manifold theory [4, 11, 16, e.g.] assures us that when $\gamma \neq 0$ there exists a three dimensional slow manifold parametrised by $\bar{u}, \bar{k}$ and $\bar{\omega}$ on which the evolution of the mean turbulent quantities is of the form (6) where now the quantities $\boldsymbol{\mu}$ are definite algebraic functions of the mean turbulent quantities $\bar{u}, \bar{k}$ and $\bar{\omega}$. We anticipate that this slow manifold persists to the physically relevant case of parameter $\gamma=1$.

Computer algebra readily constructs the slow manifold to errors $\mathcal{O}\left(\gamma^{2}\right)$ :

$$
\begin{aligned}
u(z, t)= & \bar{u}+\gamma \bar{u}\left(-\frac{2}{11}+\frac{6}{11} \zeta-\frac{3}{11} \zeta^{2}\right)+\cdots ; \\
k(z, t)= & \bar{k}+\gamma \eta \bar{u} \bar{\omega}\left(\frac{19}{176}-\frac{57}{176} \zeta+\frac{57}{352} \zeta^{2}\right) \\
& +\gamma \eta^{2} \bar{\omega}\left(-\frac{1}{24}+\frac{1}{8} \zeta-\frac{1}{16} \zeta^{2}\right)+\cdots ;
\end{aligned}
$$




$$
\begin{aligned}
\omega(z, t)= & \bar{\omega}+\gamma \frac{\bar{u}}{\eta}\left(\frac{13122}{1243}-\frac{39366}{1243} \zeta+\frac{19683}{1243} \zeta^{2}\right) \\
& +\gamma \frac{\eta^{2} \bar{\omega}^{3}}{\bar{k}}\left(-\frac{1}{339}+\frac{1}{113} \zeta-\frac{1}{226} \zeta^{2}\right)+\cdots
\end{aligned}
$$

where the non-dimensional vertical position is $\zeta=z / \eta$. These $\mathcal{O}(\gamma)$ terms provide parabolic approximations to the vertical structure of the turbulent fields. Higher order terms in the artificial parameter $\gamma$ would give higher order polynomial approximations to the turbulent vertical structure. However, for the moment we truncate to errors $\mathcal{O}\left(\gamma^{2}\right)$ in order to keep the model as simple as possible at the expense of accuracy.

The evolution on the slow manifold Computer algebra iteration also uncovers the evolution of the mean turbulent quantities. To errors $\mathcal{O}\left(\gamma^{2}\right)$, corresponding to the slow manifold (11)-(13), but now evaluated at parameter $\gamma=1$ to obtain the physically relevant model

$$
\begin{aligned}
\frac{\partial \bar{u}}{\partial t} & =-0.55 \frac{\bar{k}}{\eta^{2} \bar{\omega}} \bar{u}+g_{x}+\cdots ; \\
\frac{\partial \bar{k}}{\partial t} & =-0.153 \bar{\omega} \bar{k}+0.162 \frac{\bar{k} \bar{u}}{\eta}+\cdots ; \\
\frac{\partial \bar{\omega}}{\partial t} & =-0.079 \bar{\omega}^{2}+15.8 \frac{\bar{k} \bar{u}}{\eta^{3} \bar{\omega}}+\cdots
\end{aligned}
$$

Figure 5 shows simulations of this system of three, coupled nonlinear ODEs for the mean flow. As shown in Figure 5: the lateral component of gravity $g_{x}$ accelerates the flow via (14); which in turn promotes growth of turblent energy $\bar{k}$ and $\bar{\omega}$ via the production terms in (15) and (16); when $\bar{k}$ and $\bar{\omega}$ become large enough the growth in the flow and in the turblence is arrested. These three ODEs summarise the dynamics of mean turbulent quantities in turbulent flows when there are no lateral variations.

The model (14)-(16) predicts uniform channel flow. Solving (14)-(16) for 


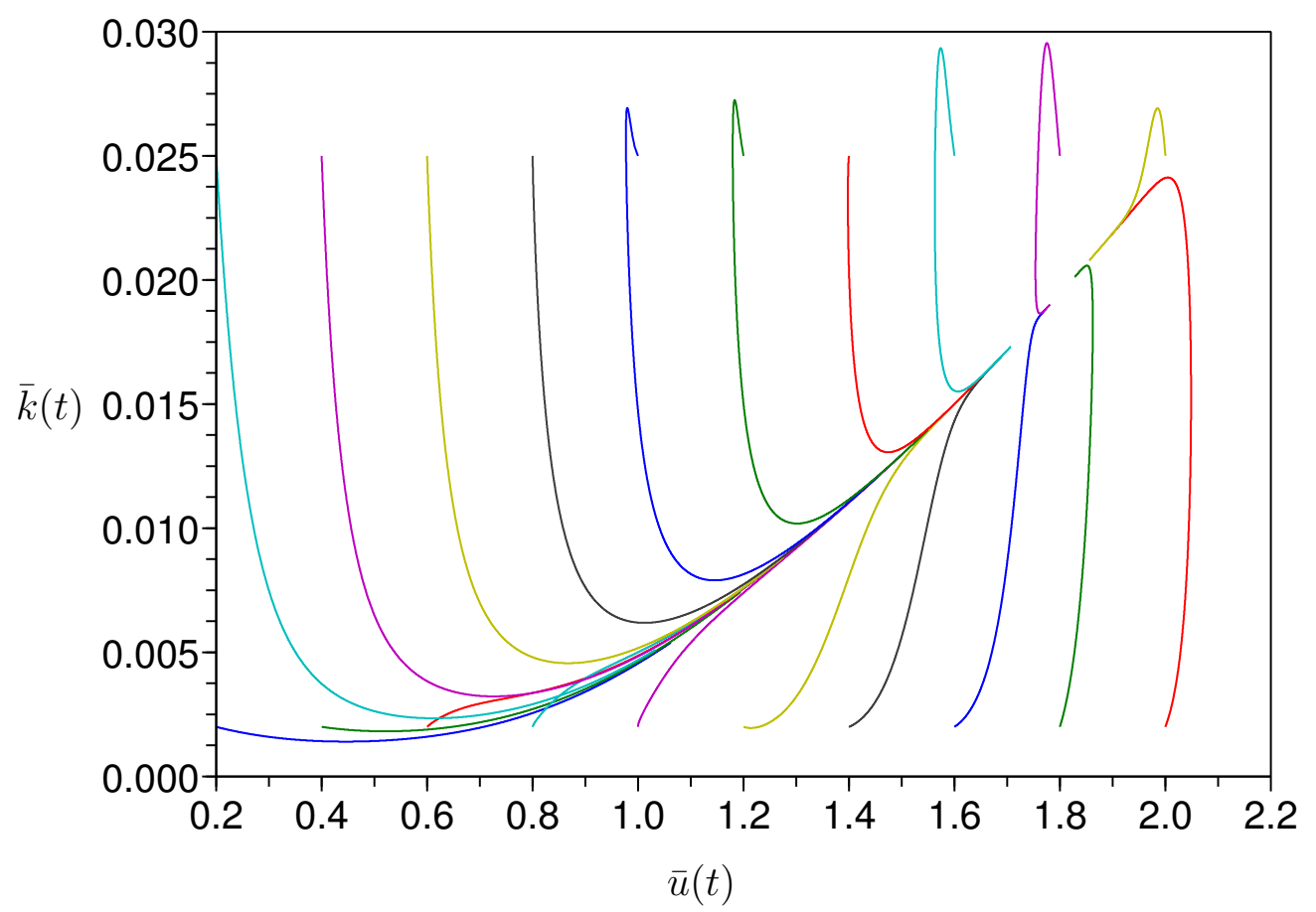

Figure 5: trajectories of turbulent flow $\bar{u}$ and turbulent energy $\bar{k}$ from many different initial conditions shows the turbulent model (14)-(16) settles evolving towards the equilibrium (17) of uniform channel flow. Here $g_{x}=.01$, $\eta=1$. 
equilibria gives

$$
\bar{u}=18.0 \sqrt{g_{x} \eta}, \quad \bar{k}=1.95 g_{x} \eta, \quad \bar{\omega}=19.2 \sqrt{g_{x} / \eta} .
$$

Simulations in Figure 5 display the evolution to this equilibrium. This equilibrium matches well the numerically determined equilibrium (7), and hence reproduces the Chezy drag law. The slow manifold model (14)-(16) appears to reasonably predict uniform channel flow; but it additionally resolves transient and other out-of-equilibrium dynamics.

For example, a turbulent flow across a horizontal plain, $g_{x}=0$, slows down by bed friction through turbulent dissipation. The model (14)-(16) predicts the power law decay of velocity $\bar{u} \sim t^{-1}$, turbulent energy $\bar{k} \sim t^{-2}$ and $\bar{\omega} \sim t^{-1}$. Numerically determining the coefficients finds two similarity solutions that may emerge in the long term decay:

$$
\begin{aligned}
\bar{u} & =.0196 \eta t^{-1}, \quad \bar{k}=24.1 \eta^{2} t^{-2}, \quad \bar{\omega}=13.1 t^{-1} ; \\
\text { and } \quad \bar{u} & =311 \eta t^{-1}, \quad \bar{k}=630 \eta^{2} t^{-2}, \quad \bar{\omega}=344 t^{-1} .
\end{aligned}
$$

Unfortunately we are not aware of any experiments to confirm either of these predicted decays. They may perhaps be observed in innudations of large flood plains.

\section{Conclusion}

This article concentrates on modelling the dynamics of turbulent flows which have no lateral variations. The reason is to understand how we can model the vertical structure of turbulent fields simultaneously with uncovering the evolution of mean turbulent quantities. However, as shown in Figure 1, interesting turbulent flows often have large lateral variations. For example, localised wave breaking generates high levels of turbulence which in turn generates pressure gradients that drive lateral flows between regions of high 
and low turbulence. Our next task is to include lateral variations to capture complex flows with horizontally variations both in depth profile and in turbulent flow properties. We will then obtain a rational model to predict flow in rivers, estuaries, floods and tsunamis.

\section{References}

[1] Paolo Blondeaux. Mechanics of coastal forms. Annu. Rev. Fluid Mech., 33:339-370, 2001. doi:10.1146/annurev.fluid.33.1.339. C574

[2] A. F. Blumberg, B. Galperin, and D. J. O'Conner. Modeling vertical structure of open-channel flows. J. Hydraulic Engineering, 118(8):1119-1134, 1992. http://cedb.asce.org/cgi/WWWdisplay.cgi?9203798. C577

[3] A. F. Blumberg and G. L. Mellor. A description of a three-dimensional coastal ocean circulatiom model. In N. S. Heaps, editor, Three-dimensional coastal ocean models, volume 118. American Geophysics Union, Washington, D.C., 1987. C577

[4] J. Carr. Applications of centre manifold theory, volume 35 of Applied Math. Sci. Springer-Verlag, 1981. C583

[5] Lars Davidson. An introduction to turbulence models. Technical Report 97/2, Chalmers University of Technology, Department of thermo and fluid dynamics, November 2003. C576

[6] J. Fredsoe and R. Deigaard. Mechanics of coastal sediment transport, volume 3 of Adv. Series on Ocean Eng. World Sci., 1992. C574

[7] Edward S. Gross, Jeffrey R. Koseff, and Stephen G. Monismith. Evaluation of advective schemes for estuarine salinity simulations. Journal of Hydraulic Engineering, 125(1):32-46, 1999. doi:10.1061/(ASCE)0733-9429(1999)125:1(32). C574 
[8] Edward S. Gross, Jeffrey R. Koseff, and Stephen G. Monismith. Three dimensional salinity simulations of south San Francisco Bay. Journal of Hydraulic Engineering, 125(11):1199-1209, 1999. doi:10.1061/(ASCE)0733-9429(1999)125:11(1199). C575, C577

[9] Andrew J. Hogg and David Pritchard. The effects of hydraulic resistance on dam-break and other shallow inertial flows. J. Fluid Mech., 501:179-212, 2004. doi:10.1017/S0022112003007468. C574, C582

[10] J. C. R. Hunt. Inland and coastal flooding: developments in prediction and prevention. Phil. Trans. R. Soc. A, 363:1475-1491, 2005. doi:10.1098/rsta.2005.1580. C574

[11] Y. A. Kuznetsov. Elements of applied bifurcation theory, volume 112 of Applied Mathematical Sciences. Springer-Verlag, 1995. C574, C583

[12] G. N. Mercer and A. J. Roberts. A complete model of shear dispersion in pipes. Jap. J. Indust. Appl. Math., 11:499-521, 1994. C575

[13] Iehisa Nezu. Open-channel flow turbulence and its research prospect in the 21st century. J. Hydraulic Engineering, page 229, April 2005. doi:10.1061/(ASCE)0733-9429(2005)131:4(229). C577

[14] A. J. Roberts. The application of centre manifold theory to the evolution of systems which vary slowly in space. J. Austral. Math. Soc. B, 29:480-500, 1988. C574

[15] A. J. Roberts. Low-dimensional models of thin film fluid dynamics. Phys. Letts. A, 212:63-72, 1996. doi:10.1016/0375-9601(96)00040-0. C574

[16] A. J. Roberts. Low-dimensional modelling of dynamical systems applied to some dissipative fluid mechanics. In Rowena Ball and Nail Akhmediev, editors, Nonlinear dynamics from lasers to butterflies, 
volume 1 of Lecture Notes in Complex Systems, chapter 7, pages 257-313. World Scientific, 2003. C583

[17] A. J. Roberts. Shear dispersion along circular pipes is affected by bends, but the torsion of the pipe is negligible. SIAM J. Applied Dynamical Systems, 3:433-462, 2004. http://epubs.siam.org/sam-bin/dbq/article/60088. C575

[18] David C. Wilcox. Turbulence modeling for CFD. DCW Industries Inc, La Canada, California, second edition, 1998. C576 


\section{Author addresses}

1. D. J. Georgiev, Computational Engineering and Sciences Research Centre, University of Southern Queensland, Toowoomba, Queensland 4352, Australia. mailto:georgiev@usq.edu.au

2. A. J. Roberts, Computational Engineering and Sciences Research Centre, University of Southern Queensland, Toowoomba, Queensland 4352, Australia. mailto:aroberts@usq.edu.au

3. D. V. Strunin, Computational Engineering and Sciences Research Centre, University of Southern Queensland, Toowoomba, Queensland 4352, Australia. mailto: strunin@usq. edu.au 